\title{
Conservative treatment of upper urinary tract carcinoma: Long-term results
}

\author{
Andrea Orosa Andrada, MD; Inés Laso García, MD; Fernando Arias Fúnez, MD; Francisco Donis Canet, MD; \\ Gemma Duque Ruiz, MD; Victoria Gómez Dos Santos, MD; Francisco Javier Burgos Revilla, MD
}

Department of Urology, Ramón y Cajal University Hospital, Madrid, Spain

Cite as: Can Urol Assoc J 2017;11 (7):E291-6. http://dx.doi.org/10.5489/cuaj.4173 Published online July 11, 2017

\section{Abstract}

Introduction: We sought to assess the long-term results of conservative treatment for upper urinary tract carcinoma (UUTC) with regard to tumour recurrence and preservation of renal unit.

Methods: From October 1987 to January 2014, 65 patients (median age 68 years) were diagnosed with UUTC and underwent endoscopic and open surgical techniques. Thirteen patients had bilateral disease and one had a single kidney. The primary approach was endoscopic in 37 reno-ureteral units (20 percutaneous resections, 17 ureteroscopies ). Open surgery was performed in 19 cases. A total of 20 patients received mitomycin $\mathrm{C}$.

Results: Superficial stage pTa or T1 was noted in 37 patients, infiltrating stage pT2 and pT3 in seven and inverted papilloma in one. The stage of the tumour was impossible to classify in 20. With a median followup of 75.12 months (interquartile range [IQR] 144.71-17.41), the kidney preservation, recurrence, specific survival, and global survival rates were 78.5\% (51/65), 40.0\% (26/65), $92.3 \%(60 / 65)$, and $69.2 \%(45 / 65)$, respectively. From the patients who had recurrence, 15 were salvaged with radical nephroureterectomy (RNU). The bladder tumour recurrence rate after the surgery was $30.76 \%$ (20/65). At the end of the followup, five patients had died of UUTC progression and 16 from other causes. Postoperative complications included one case of fistula, one case of stricture, and one case of nephrectomy due to bleeding.

Conclusions: In selected cases, conservative management is a safe and feasible alternative to RNU, with the advantage of renal unit preservation.

\section{Introduction}

Upper urinary tract carcinoma (UUTC) is uncommon and accounts for $5-10 \%$ of all urothelial carcinomas. ${ }^{1,2}$ Radical nephroureterectomy (RNU) with excision of the bladder cuff is the gold standard treatment for localized disease, regardless of the location of the tumour in the upper urinary tract.

An interest in the conservative surgery, including endoscopic ablation and segmental resection, has emerged in recent years as an alternative for patients with UUTC and renal insufficiency, solitary functional kidney, comorbidities, or bilateral tumour, accounting for approximately $2-5 \%$ of UUTC patients. ${ }^{3}$

The elective cases for nephron-sparing surgery are considered in patients with low-grade, low-stage, unifocal, small tumours. In addition, the patient has to accept close followup because of the risk of recurrence, which is the main disadvantage of this treatment. ${ }^{4}$

After RNU, the presence of a solitary kidney can lead to a renal insufficiency stage, which is associated with an increase in cardiovascular events and, therefore, in overall mortality. ${ }^{4}$ It is well-documented that glomerular filtration rate decreases after RNU, with a mean of $24 \%$, according to the outcomes of a recent multicentre analysis by Kaag et al. ${ }^{5}$ These changes in renal function may affect eligibility for adjuvant cisplatin based-therapy. ${ }^{5}$

Moreover, multifocal recurrence of UUTC in the contralateral side has renewed the interest in conservative treatment in elective cases. Furthermore, retrospective analysis suggests that patients with low-grade and low-stage disease benefit from a nephron-sparing surgery with similar outcomes at five years to those achieved with RNU. ${ }^{6}$

The aim of this study is to assess the long-term oncological results in terms of recurrence, specific survival, and global survival, as well as the preservation of the renal unit in the conservative treatment of UUTC.

\section{Methods}

From October 1987 to January 2014, 65 patients were diagnosed with UUTC and treated with conservative techniques, including endoscopic approach and open surgery. Most $(87.7 \%[57 / 65])$ of the patients were men, $43.1 \%(28 / 65)$ were smokers, and $33.8 \%(22 / 65)$ had a history of bladder tumour. The median age was 68 years (range 40-84). Preoperative workup included intravenous pyelography, computed tomography (CT) scan, urinary cytology, and a general medical patient evaluation. In the most recent cases, the diagnosis was completed with a ureteroscopy (URS) and biopsy, as noted in the main guidelines. ${ }^{7}$ 
Andrada et al.

The majority of the patients $(n=50)$ had a normal contralateral kidney, representing an elective indication for conservative treatment. Thirteen patients had bilateral tumour, one had single kidney, and another one suffered from important comorbidities. Patients with UUTC with high risk of local invasion or patients refusing intensive endoscopic followup were excluded. Tumour features are summarized in Table 1.

Seventeen URS were performed and the tumour was destroyed with Holmium laser in 11 cases and electroresection in six cases. A percutaneous approach was selected in 20 patients. Nineteen patients underwent open procedures, the most frequent being segmental ureterectomy, with 16 cases performed. The rest comprised of two pyelectomies and one bilateral partial nephrectomy.

Regarding the patients with bilateral tumour $(n=13)$, eight were treated with radical RNU and contralateral conservative approach (four percutaneous resections, one pyelectomy, three ureterectomies). The rest of the patients $(n=5)$ with bilateral tumours underwent percutaneous resections in three cases, one went through a bilateral partial nephrectomy intervention, and another was treated with percutaneous approach and contralateral URS.

After the intervention, 20 patients received adjuvant therapy with mitomycin C. The aim of this therapy was prophylactic in an attempt to minimize recurrences. The treatment was administered via nephrostomy tube in 18 cases after a percutaneous approach, and the remaining two received mitomycin $C$ via ureteral catheter.

The choice of the technique was based on the size and location of the tumour, as well as the features of the patient. The median tumour size was $10 \mathrm{~mm}$ (range 5-30). Tumours chosen for URS treatment were those with low volume and located in the ureter or upper renal cavities.

In cases of larger tumours or those located in the lower caliceal system, inaccessible, or difficult to manage with URS, the percutaneous approach was the option. Ureterectomy was indicated for tumours in the distal and medium ureter, which could not be removed completely by endoscopic procedures.

The followup protocol of the patients was heterogeneous, as the study includes a historic case series with a long sur-

\begin{tabular}{lccc}
\hline \multicolumn{4}{l}{ Table 1. Tumour features and surgical indications } \\
\hline Indication & $\mathbf{n}$ & Tumour location & $\mathbf{n}$ \\
\hline General & 1 & Kidney & \\
Solitary & Calyceal & 3 \\
Bilateral & 13 & Renal pelvis & 22 \\
Relative (comorbidity) & 1 & Ureter & \\
& & Lumbar & 6 \\
Elective & 50 & Sacral & 1 \\
& & Pelvic & 22 \\
Total & & Various locations & 11 \\
\hline
\end{tabular}

veillance time. The patients who were treated during the 90s were followed with ultrasounds, intravenous urography, urinary cytology, and cystoscopy every three months during the first year, and then every six months thereafter. Currently, we follow the indications recommended by the European guidelines. The surveillance regimen is based on urinary cytology and CT urography at three and six months, and yearly thereafter, as well as cystocopy, URS, and cytology in situ at three and six months, then every six months for two years, and finally, yearly.

The primary endpoints were the recurrence in the upper urinary tract and bladder, as well as renal unit survival. Overall survival, disease-specific survival, UUTC and bladder recurrence-free survival, and renal unit survival were assessed by Kaplan-Meier survival curves. The results according to the indication (elective vs. imperative) were analyzed. Logistic univariate and Cox regression analysis were performed to analyze potential risk factors for UUTC recurrence, including multifocality, tumour grade, tumour size, previous bladder cancer, surgical approach, and tobacco use. SPSS ${ }^{\circledR}$ version 20 was used for all statistical analyses.

\section{Results}

The median followup was 75.12 months (interquartile range [IQR] 144.71-17.41). [The histology was available in 60 cases. It turned out to be malignant in all cases except one case of a benign tumour (inverted papilloma). In five cases, the histology was unknown. The pathology after conservative treatment is summarized in Table 2. Due to the malignant potential of the inverted papilloma, it has been included in all analyses. The postoperative complications are summarized in Table 3.

At the end of followup, overall survival and disease-specific survival rates were $69.2 \%(45 / 65)$ and $92.30 \%$ (60/65), respectively. The related Kaplan-Meier curves are shown in Fig. 1.

Among the 65 patients, five died of UUTC. Four of these patients had recurrence after endoscopic management, three of whom were salvaged with RNU and the other was rejected from surgery due to comorbidities. The final patient had rapid metastases and died after the conservative treatment.

With a followup median of 75.12 months, UUTC recurrence rate was $4.0 \%(26 / 65)$. Among patients with recurrence, one was contralateral and another was bilateral. The median time to recurrence was 136.28 months (95\% confidence interval $[\mathrm{Cl}]$ 100.71-171.847).

Bladder recurrence accounted for $30.8 \%(20 / 65)$ at the end of followup. The median time was not achieved. The $75^{\text {th }}$ percentile was 43.47 months, when $25 \%$ of the patients had a bladder recurrence. Two radical cystectomies were necessary. Fig. 2 shows the Kaplan-Meier curves for upper urinary tract and bladder recurrence survival. 


\begin{tabular}{lc}
\hline Table 2. Pathology & $\mathbf{n}$ \\
\hline Final pathology & \\
\hline Benign tumours & 1 \\
Inverted papilloma & \\
pTa & 10 \\
Grade 1 & 7 \\
Grade 2 & 1 \\
Grade 3 & \\
pT1 & 8 \\
Grade 1 & 10 \\
Grade 2 & 1 \\
Grade 3 & \\
pT2 & \\
Grade 2 & 2 \\
Grade 3 & 3 \\
pT3 & \\
Grade 2 & \\
Grade 3 & 1 \\
pTx & 1 \\
Grade 1 & \\
Grade 2 & 3 \\
Grade 3 & \\
Unknown & 3 \\
Unknown & 3 \\
Total & 55 \\
\hline
\end{tabular}

RNU was the salvage therapy for UUTR in 14 cases; ureterectomy was performed in three cases. The rest of recurrences were treated through an endoscopic approach. Estimated renal unit survival rate was 78.5\% (51/65) (Fig. 3).

Multifocality, history of bladder cancer, tobacco use, tumour grade, and tumour size were not statistically associated to recurrence. Nevertheless, we analyzed the influence of the type of surgery (ureterectomy vs. endoscopic approach) and $48 \%$ of the patients treated with endoscopic techniques had recurrences vs. $18 \%$ of the patients treated with open surgery $(p=0.027)$ (Fig. 4).

The difference between the patients with an imperative indication $(n=15)$ and those with an elective indication $(n=50)$ in terms of recurrence, renal unit survival, overall and disease-specific survival was not statistically significant.

\section{Discussion}

This study highlights that selected patients could benefit from conservative treatment for bladder cancer and UUTC recurrence in terms of efficacy and disease-specific mortality.

Most of the recent literature on UUTC are retrospective case series, with a limited number of patients and a short followup. Moreover, there are no randomized trials comparing conservative treatment and RNU.

Simhan et al have published a retrospective review using a national database (Surveillance, Epidemiology and End Results [SEER]). ${ }^{3}$ The review included 1227 patients diagnosed with UUTC and treated with RNU or conservative treatment, with a median followup of 61 months; ${ }^{3}$ however, the study has limitations inherent to a national database in terms of patient selection and probable bias.

The current study reports a similar sample size to other papers published, ${ }^{8-13}$ but with a longer followup. This allows us to assess the long -term effects after conservative treatment. On the other hand, we have included the ureterectomy as a surgical conservative option according to the current European guidelines, ${ }^{7}$ unlike the majority of published articles, which are limited to endoscopic surgery.

Nonetheless, the limitation to this series is its retrospective nature. Also, the long-term followup involves a heterogeneous followup protocol of the patients, with difficulties in the diagnosis of low-grade recurrences in the older cases. Further, because this series has a long-term followup, indications for radical surgery may have changed.

Still, our results are similar to other studies published. The UUTC recurrence is the main concern with this type of conservative treatment. The recurrence rate is variable depending on the different studies (20-85\%). ${ }^{14}$ In a recent systematic review, Cutress et al estimated an UUTC recurrence after URS and percutaneous resection of $52 \%$ and $37 \%$, respectively, with a median followup of five years. ${ }^{9}$ Stratifying by tumour grade, the recurrence rate is greater the higher the tumour grade. ${ }^{9}$

Although in our series we did not find a statistical association between recurrence and tumour grade, prior history of bladder tumours, tumour size, and multifocality, this association has been reported in the literature ${ }^{11,15,16}$ and establishes the mainstays of conservative treatment. ${ }^{7}$ However, in this

\begin{tabular}{|c|c|c|c|c|}
\hline Complication & Surgical technique & Number of complications & Clavien-Dindo & Treatment \\
\hline Bleeding & Percutaneous resection & 1 & $\mathrm{IIIb}$ & Nephrectomy \\
\hline Urinary fistula & Percutaneous resection & 1 & Illa & Ureteral catheter \\
\hline Ureteral stricture & Ureterectomy & 1 & IIlb & Ureteral re-implantation \\
\hline \multirow{2}{*}{ Urinary tract infection } & URS & 1 & II & Antibiotic \\
\hline & Percutaneous resection & 2 & II & Antibiotic \\
\hline
\end{tabular}




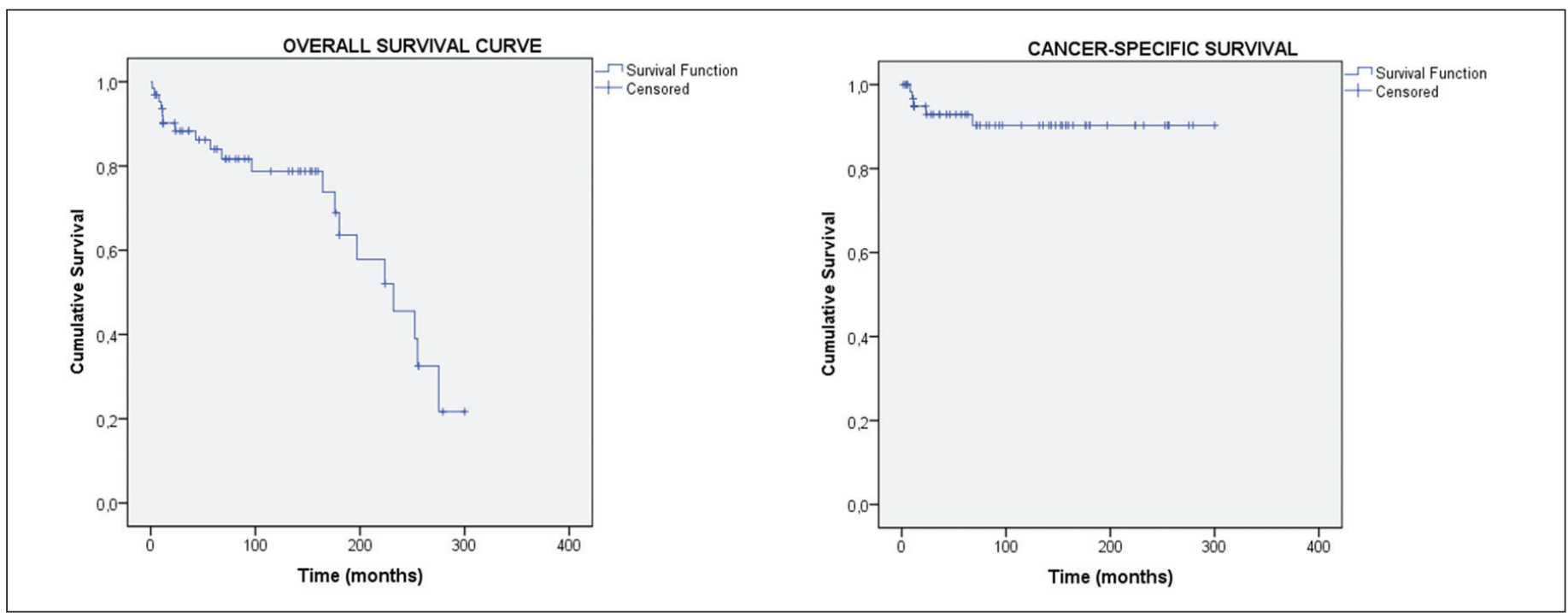

Fig. 1. The overall survival curve shows that $50 \%$ of patients are alive after a median of 232.05 months. Five patients died due to upper urinary tract cancer during the first 75 months.

study, the patients treated by ureterectomy had fewer recurrences in the upper urinary tract than patients treated by endoscopic approach. This is probably because the ureterectomy allows a more radical surgery than endoscopic treatment.

As recurrence is common and recurrence rate is greater the higher the tumour grade, the diagnosis must be precise for better results following a conservative approach. CT urography is the imaging technique with the highest accuracy for UUTC; however, the sensitivity and specificity may vary depending on the technique used and the tumour size. ${ }^{7}$

A biopsy should be done when performing the flexible URS to determine the tumour grade. This allows better precision and the possibility of selecting the best treatment in every patient.
The interest in increasing the accuracy of the diagnosis has led Brien et al to design a forecast model based on hydronephrosis, urinary cytology. and the grade of the disease in URS biopsy. Such knowledge may impact surgery choice or the need for perioperative chemotherapy regimens. ${ }^{17}$

In patients considered for conservative treatment, the value of the URS biopsy in stratifying the tumour grade is well-established by the guidelines; however, the diagnostic value is controversial. This is due to the fact that, despite the new technology available, the ability to obtain tissue is still limited by different variables. This results in a small volume of biopsy, commonly without muscle sample. Rojas et al published a review of 137 URS biopsies in 81 patients,
A

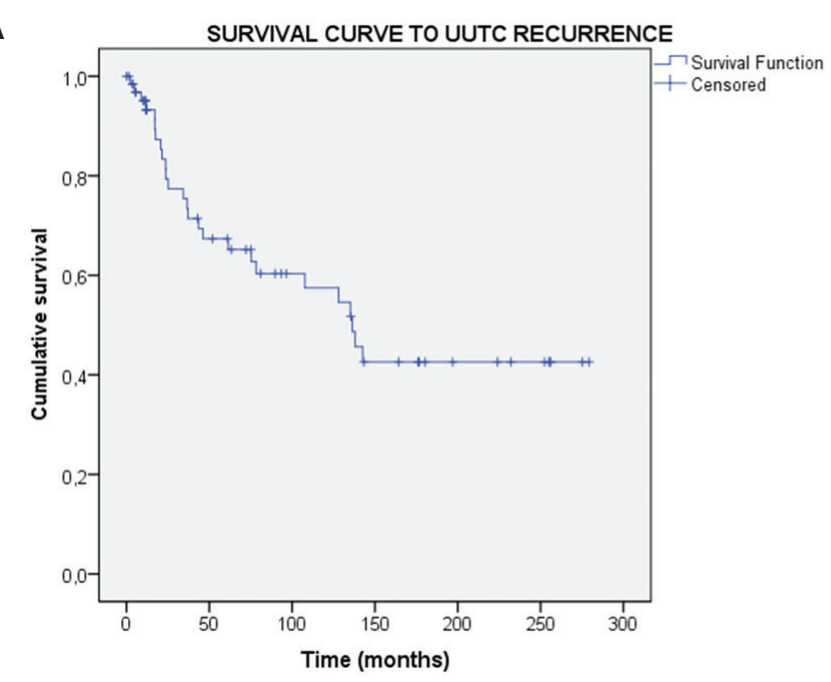

B

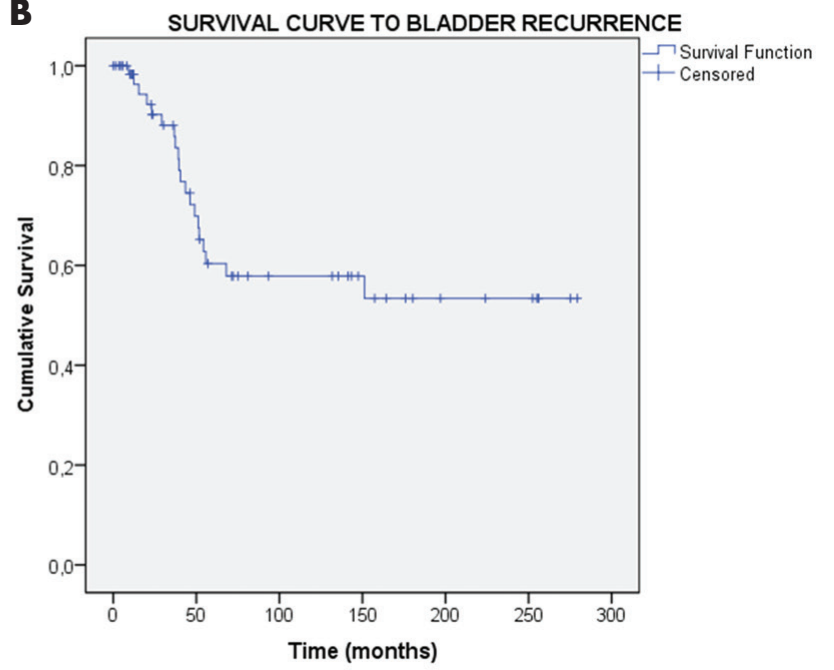

Fig. 2. The survival curves of (A) upper urinary tract cancer recurrence; and (B) bladder cancer recurrence. 


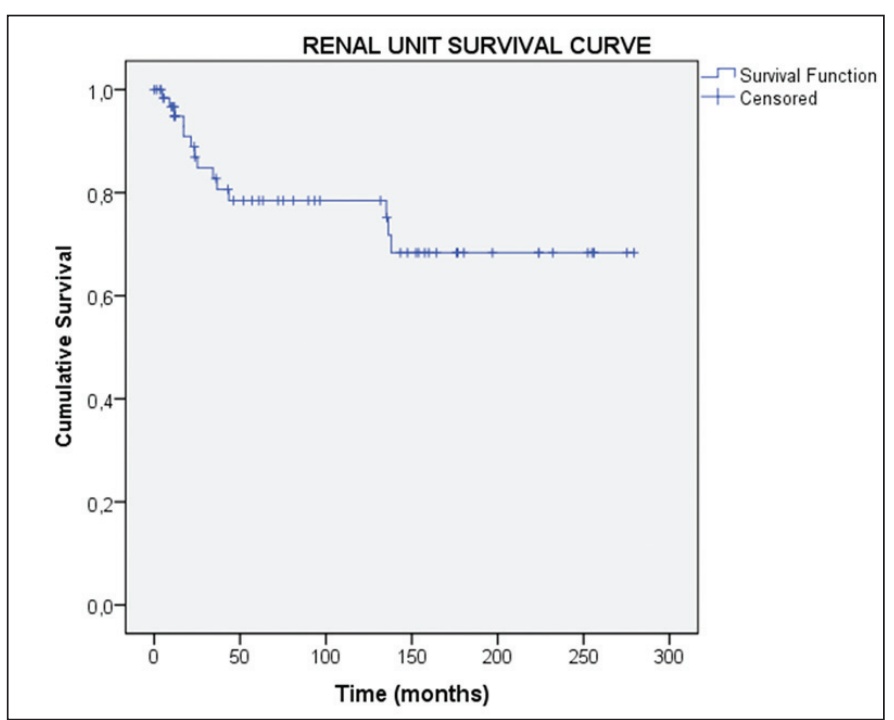

Fig. 3. Renal unit survival curve. The 75th percentile was 136.23 months.

of which 54 underwent RNU. The grade concordance was $92 \%$; however, the stage concordance was only $43 \% .^{18}$ Clements et al defined a predictive accuracy of high-grade and muscle-invasive disease of $90 \%$ and $60 \%$, respectively. ${ }^{19}$ Similarly, other authors observed a greater than expected rate of tumour upgrading and invasive disease, particularly in those with low-grade tumours at biopsy. ${ }^{14}$

It is important to reiterate that our study includes ureterectomy as part of conservative treatment. In addition, we observed that patients treated with this technique had a lower rate of recurrence than patients undergoing endoscopic surgery. This finding may be explained because ureterectomy allows the complete resection of the tumour, making this procedure a feasible alternative in selected cases of high-risk or muscle-invasive disease. ${ }^{20,21}$

Another reason why nephron-sparing surgery is preferable to RNU in selected patients is the lower complication rate. The risk of perioperative complications after an URS is about $14 \%$, with a probability of a stricture during the followup of about $11 \%$. Meanwhile, percutaneous resection has a much higher risk of complication $(27 \%) .{ }^{9}$ Concern regarding tract seeding after a percutaneous approach is highly unlikely, standing at $0.75 \%$ in the most experienced institutes. ${ }^{22}$ Moreover, renal unit preservation, which in this study reaches nearly $80 \%$ after a mean followup of 75 months, allows us to cope in better conditions with the risk of a contralateral tumour $(6 \%) .{ }^{23}$

On the other hand, the decrease of glomerular filtration rate following RNU and therefore the risk of developing complications such as chronic kidney disease, may affect eligibility for adjuvant cisplatin-based therapies, particularly in elderly patients. ${ }^{4}$

Furthermore, if we bear in mind that salvage RNU of the recurrences after conservative treatment provides comparable outcomes to immediate RNU in patients with low-

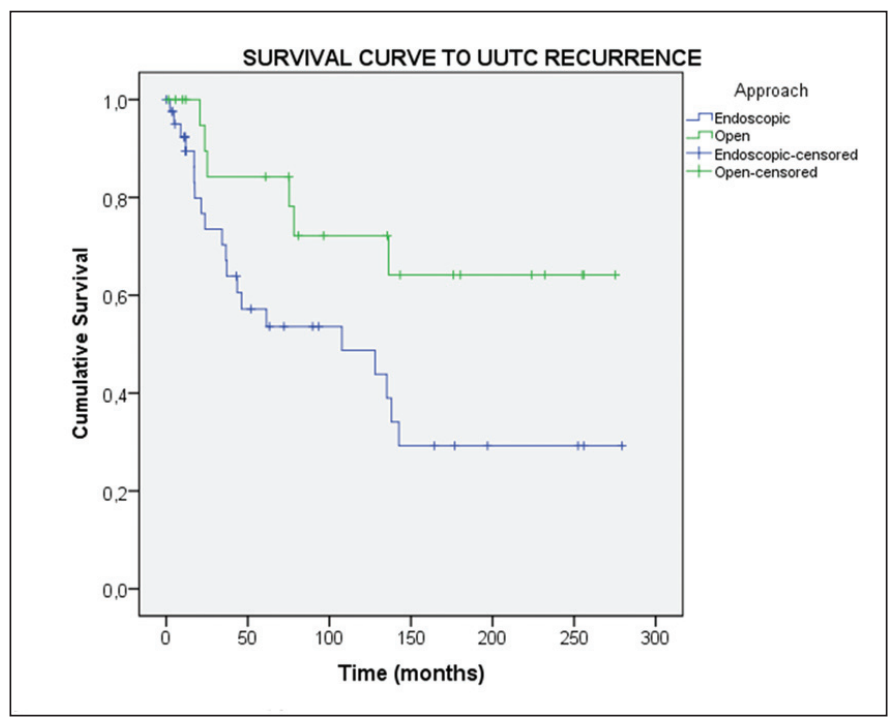

Fig. 4. Patients treated with open procedures had fewer recurrences than patients treated with endoscopic approach.

grade disease, ${ }^{24-26}$ conservative treatment becomes a safe and a feasible option in selected cases.

\section{Conclusion}

Conservative treatment of UUTC is a therapeutic alternative that, in selected cases, may provide a feasible alternative to $\mathrm{RNU}$, but with the added advantage of renal unit preservation. The long-term followup of this study allow us to better understand the natural history of the disease after conservative management, potentially leading to the establishment of specific indications for its use in order to obtain the greatest benefit for our patients.

Competing interests: Dr. Garcia has received payment from Bayer and has participated in clinical trials supported by Soria Natural. Dr. Dos Santos and Dr. Revilla have participated in prostate cancer clinical trials supported by Medivation. The remaining authors report no competing personal or financial interests.

This paper has been peer-reviewed.

\section{References}

1. Siegel R, Naishadham D, Jemal A. Cancer statistics, 2012. CA Cancer J 2015;62:10-29. https://doi.org/10.3322/caac.20138

2. Munoz JJ, Ellison LM. Upper tract urothelial neoplasms: Incidence and survival during the last two decades. J Urol 2000;164:1523-5. https://doi.org/10.1016/S0022-5347(05)67019-X

3. Simhan J, Smaldone MC, Egleston BL, et al. Nephron-sparing management vs. radical nephroureterectomy for low- or moderate-grade, low-stage upper tract urothelial carcinoma. BJU Int 2014;114: 216-20. https://doi.org/10.1111/bju.12341

4. Kaag MG, $0^{\prime}$ Malley RL, $0^{\prime}$ Malley $P$, et al. Changes in renal function following nephroureterectomy may affect the use of perioperative chemotherapy. Eur. Urol 2010;58:581-7. hitps://doi.org/10.1016/i. eururo.2010.06.029 
Andrada et al.

5. Bravo Fernández I, García Cardoso JV, Pérez Ortiz G, et al. Carcinoma urothelial ureteral bilateral sincrónico. Actas Urológicas Espa-olas 2006;30:335-9. htrps://doi.org/10.1016/S0210-4806(06)73451-9

6. Cutress ML, Stewart G.D, Tudor ECG, et al. Endoscopic vs. laparoscopic management of non-invasive upper tract urothelial carcinoma: 20-year, single-centre experience. J Urol 2013;189:2054-60. https://doi.org/10.1016/i.juro.2012.12.006

7. Rouprêt $M$, Babjuk $M$, Compérat $E$, et al. European guidelines on upper tract urothelial carcinomas: 2013 update. Eur Urol 2013;63:1059-71. htrps://doi.org/10.1016/i.eururo.2013.03.032

8. Cutress ML, Stewart GD, Wells-Cole $S$, et al. Long-term endoscopic management of upper tract urothelial carcinoma: 20-year, single-centre experience. BJU Int 2012;110:1608-17. https://doi.org/10.1111/ j.1464-410X.2012.11169.x

9. Cutress ML, Stewart GD, Zakikhani P, et al. Ureteroscopic and percutaneous management of upper tract urothelial carcinoma (UTUC): Systematic review. BJU Int 2012;1 10:614-28. https://doi.org/10.1111/ j.1464-410X.2012.11068.x

10. Daneshmand S, Quek ML, Huffman JL. Endoscopic management of upper urinary tract transitional cell carcinoma: Long-term experience. Cancer 2003;98:55-60. htrps://doi.org/10.1002/cncr.11446

11. Deligne $E$, Colombel $M$, Badet L, et al. Conservative management of upper urinary tract tumours. Eur Urol 2002; 42:43-8. https://doi.org/10.1016/50302-2838(02)00220-8

12. Grasso $\mathrm{M}$, Fishman Al, Cohen J, et al. Ureteroscopic and extirpative treatment of upper urinary tract urothelial carcinoma: A 15-year comprehensive review of 160 consecutive patients. BJU Int 2012;110:1618-26. https://doi.org/10.1111/j.1464-410X.2012.11066.x

13. Martínez-Pieiro JA, García Matres MJ, Martínez-Pieiro L. Endourological treatment of upper tract urothelia carcinomas: Analysis of a series of 59 tumours. J Urol 1996;156:377-85. https://doi.org/10.1016/ S0022-5347(01)65854-3

14. Wang JK, Tollefson MK, Krambeck AE, et al. High rate of pathological upgrading at nephroureterectomy for upper tract urothelial carcinoma. Urology 2012;79:615-9. https://doi.org/10.1016/i.urology.2011.11.049

15. Rouprêt $M$, Hupertan V, Traxer 0 , et al. Comparison of open nephroureterectomy and ureteroscopic and percutaneous management of upper urinary tract transitional cell carcinoma. Urology 2006;67:1181-7. hitps://doi.org/10.1016/i.urology.2005.12.034

16. Iborra I, Solsona E, Casanova J, et al. Conservative elective treatment of upper urinary tract tumours: A multivariate analysis of prognostic factors for recurrence and progression. J Urol 2003;169:82-5. https://doi.org/10.1016/S0022-5347(05)64041-4

17. Brien JC, Shariat SF, Herman MP, et al. Preoperative hydronephrosis, ureteroscopic biopsy grade, and urinary cytology can improve prediction of advanced upper tract urothelial carcinoma. J Urol 2010;184:69-73. https://doi.org/10.1016/i.juro.2010.03.030
18. Rojas CP, Castle SM, Llanos CA, et al. Low biopsy volume in ureteroscopy does not affect tumour biopsy grading in upper tract urothelial carcinoma. Urol Oncol 2013;31:1696-700. https://doi.org/10.1016/i. urolonc.2012.05.010

19. Clements T, Messer JC, Terrell JD, et al. High-grade ureteroscopic biopsy is associated with advanced pathology of upper-tract urothelial carcinoma tumours at definitive surgical resection. J Endourol 2012;26:398402. https://doi.org/10.1089/end.2011.0426

20. Colin P, Ouzzane A, Pignot $G$, et al. Comparison of oncological outcomes after segmental ureterectomy or radical nephroureterectomy in urothelial carcinomas of the upper urinary tract: Results from a large French multicentre study. BJU Int 2012;110:1134-41. https://doi.org/10.1111/i.1464-410X.2012.10960.x

21. Jeldres C, Lughezzani $G$, Sun $M$, et al. Segmental ureterectomy can safely be performed in patients with transitional cell carcinoma of the ureter. J Urol 2010;183:1324-9. https://doi.org/10.1016/i. juro.2009.12.018

22. Rastinehad AR, Ost MC, Vanderbrink BA, et al. A 20-year experience with percutaneous resection of upper tract transitional carcinoma: Is there an oncological benefit with adjuvant bacillus Calmette Guerin therapy? Urology 2009;73:27-31. https://doi.org/10.1016/i.urology.2008.06.026

23. Novara G, De Marco V, Dalpiaz 0 , et al. Independent predictors of contralateral metachronous upper urinary tract transitional cell carcinoma after nephroureterectomy: Multi-institutional dataset from three European centres. Int J Urol 2009;16:187-91. https://doi.org/10.1111/i.1442-2042.2008.02201.x

24. Boorijan $\mathrm{S}, \mathrm{Ng} \mathrm{C}$, Munver R, et al. Impact of delay to nephroureterectomy for patients undergoing ureteroscopic biopsy and laser tumour ablation of upper tract transitional cell carcinoma. Urology 2005;66:283-7. https://doi.org/10.1016/j.urology.2005.02.022

25. Lucas $S M$, Svatek RS, Olgin $G$, et al. Conservative management in selected patients with upper tract urothelial carcinoma compares favourably with early radical surgery. BJU Int 2008;102:172-6. https://doi.org/10.1111/i.1464-410X.2008.07535.x

26. Seisen T, Peyronnet B, Dominguez-Escrig JL, et al. Oncological outcomes of kidney-sparing surgery vs. radical nephrourecterectomy for upper tract urothelial carcinoma: A systematic review by the EAU Non-muscleinvasive Bladder Cancer Guidelines Panel. Eur Urol 2016;70:1052-68. https://doi.org/10.1016/i. eururo.2016.07.014

Correspondence: Dr. Andrea Orosa-Andrada; Department of Urology, Ramón y Cajal University Hospital, Madrid, Spain; andreaorosa@hotmail.com 